\title{
Traumatic lung herniation
}

\author{
Mathieu Martin, MD • Fabrice Cook, MD • Bouziane Ait Mamar, MD • \\ Gilles Dhonneur, MD, PhD
}

Received: 1 December 2015/Revised: 7 December 2015/Accepted: 14 December 2015/Published online: 21 December 2015

(C) Canadian Anesthesiologists' Society 2015

Traumatic lung herniation is a rare diagnosis, and literature on its optimal therapeutic management (i.e., medical vs surgical) is scarce. We present the case of a 53-yr-old man admitted to our trauma centre with blunt chest trauma after being crushed by the car he was repairing. An initial chest computed tomography (CT) scan on admission to the intensive care unit (ICU) revealed bilateral lung contusions, small hemopneumothorax, fractured sternum, and multiple bilateral rib fractures (with a left radiologically flail segment) associated with intense subcutaneous emphysema. As there was no clinically obvious flail segment with spontaneous ventilation, a conservative approach was used that included attempts to optimize analgesia, perform chest physiotherapy, and supply nasal high-flow oxygen therapy to improve the patient's oxygenation and comfort. The small hemopneumothorax did not necessitate placement of a chest tube.

However, $36 \mathrm{hr}$ after ICU admission, tracheal intubation was required because of respiratory failure partly resulting from retained pulmonary secretions and inadequate analgesia, both of which prevented efficient chest physiotherapy. After intubation, the patient was sedated and paralyzed, and positive-pressure ventilation was initiated. During subsequent weaning from mechanical ventilation, a change in pulmonary mechanics was noted, including non-synchronized inspiratory efforts associated

M. Martin, MD ( $₫)$ · F. Cook, MD - B. Ait Mamar, MD .

G. Dhonneur, $\mathrm{MD}, \mathrm{PhD}$

Surgical Intensive Care Unit - Trauma Center, Department of Anaesthesiology and Critical Care Medicine, Paris-Est Créteil

University and Assistance-Publique Hôpitaux de Paris, Henri

Mondor University Hospital, Créteil, France

e-mail: mathieu.martin@hmn.aphp.fr
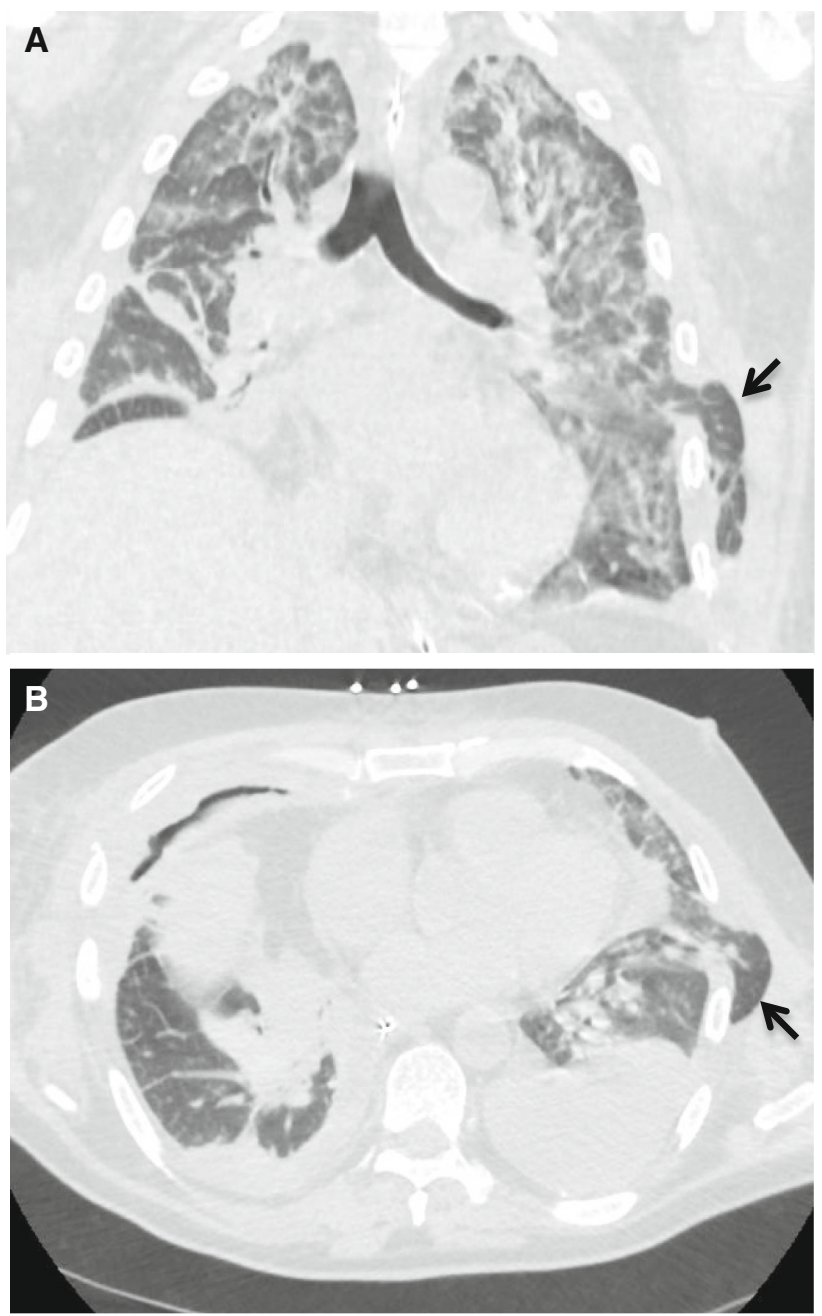

Figure Computed tomography scan of the chest following blunt chest trauma shows herniation of the left lung (black arrow) in coronal (A) and transverse cross-sectional (B) imaging planes 
with an obvious lateral left chest wall deformation. A new CT scan (Figure) revealed that the chest deformation was due to the flail chest and inflation of a large lung hernia extending outward through the intercostal muscles. The lung herniation was reduced surgically, and the flail segments were fixed. The patient was successful weaned from mechanical ventilation 16 days after the operation.

Conflicts of interest None declared. 